\title{
Research on constant attack angle flying of hypersonic aircraft Guoqiang Liang ${ }^{a}$ and Jianhong Shi
}

Department of control engineering, Naval aeronautical and astronautical University

Yanti, 264001

aliangguoqiang1024@126.com

Keywords: hypersonic aircraft; stability; control; free flying; numerical simulation

\begin{abstract}
A kind of imaginary virtual flying with numerical simulation is used to testify the rightness of hypersonic aircraft with air dynamic coefficients. Since the attack angle is the most important factor that affect the stability of the hypersonic or supersonic aircraft, so a kind of virtual flying with constant attack angle is proposed to do the open loop numerical simulation to check the air coefficients of system model. This kind of testing method is very useful for situations that the rightness of the aircraft system model is not guaranteed, especially for complex flying systems. So this new testing method is very meaningful and useful for hypersonic engineering.
\end{abstract}

\section{Introduction}

The validity and accuracy of hypersonic model is the prerequisite that carries out the hypersonic research $^{[1-4]}$. At present, the model is in a non-public status. And the united states and abroad discloses several types of hypersonic model based on different methods, but the accuracy and reasonableness of its aerodynamic data can not be guaranteed. Therefore, this article verifies the correctness of the model by the research of a series of specific simple state flight digital simulation test in connection with hypersonic vehicle longitudinal motion model published by the U.S. air force research laboratory ${ }^{[5-8]}$. At the same time, the difference between the change speed of attack angle and traditional non hypersonic aircraft are observed by simulation results. In a variety of typical simple state flight numeric simulation, it is very easy to do, but it is not easy for hypersonic vehicle, even if it is stable flight $0.5 \mathrm{~s}$, if the model is unreasonable, but also more difficult. Main reason is that the coupling relationship between elastic body shape and attack angle. In addition that the coupling between the engine and attack angle has a greater impact for stable run smoothly of flight digital simulation.

\section{Model Description}

Considering the elastic shape structure, a kind of pitch channel hypersonic aircraft model built according to Lagrange equation is released by USA air force as followed:

$$
\begin{gathered}
\dot{V}=\frac{T \cos \alpha-D}{m}-g \sin \gamma \\
\dot{\phi}=-2 \varsigma \omega_{n} \phi-\omega_{n}^{2} \phi+\omega_{n}^{2} \phi_{c} \\
\dot{\gamma}=\frac{L+T \sin \alpha}{m V}-\frac{g \cos \gamma}{V} \\
\dot{\alpha}=q-\dot{\gamma} \\
\dot{q}=\frac{M}{I} \\
\dot{h}=V \sin \gamma \\
\ddot{\eta}_{i}=-2 \varepsilon_{m} \omega_{m i} \dot{\eta}_{i}-\omega_{m i}^{2} \eta_{i}+N_{i}
\end{gathered}
$$


Where

$$
\begin{gathered}
C_{D}=C_{D}^{\alpha^{2}} \alpha^{2}+C_{D}^{\alpha} \alpha+C_{D}^{\delta_{e}^{2}} \delta_{e}^{2}+C_{D}^{\delta_{e}} \delta_{e}+C_{D}^{0}+C_{D}^{\delta_{c}^{2}} \delta_{c}^{2}+C_{D}^{\delta_{c}} \delta_{c}+C_{D}^{\eta} \eta \\
=5.8224 \alpha^{2}-0.0045315 \alpha+0.81993 \delta_{e}^{2}+0.00027699 \delta_{e} \\
+0.01013+0.7186 \delta_{c}^{2}+0.00038522 \delta_{c} \\
+5.346^{*} 10^{-2} \eta_{1}+5.346 * 10^{-2} \eta_{2}+0.543 \eta_{3} \\
C_{T \phi}=C_{T \phi}^{\alpha^{3}} \alpha^{3}+C_{T \phi}^{\alpha^{2}} \alpha^{2}+C_{T \phi}^{\alpha} \alpha+C_{T \phi}^{0} \\
=15.436 \alpha^{3}+6.2324 \alpha^{2}+7,9436 \alpha+0.5887 \\
C_{T}=C_{T}^{\alpha^{3}} \alpha^{3}+C_{T}^{\alpha^{2}} \alpha^{2}+C_{T}^{\alpha} \alpha+C_{T}^{0} \\
=10.936 \alpha^{3}+8.438 \alpha^{2}+6.564 \alpha+6.960 \\
\quad L=\bar{q} S C_{L}, \\
C_{L}=C_{L}^{\alpha} \alpha+C_{L}^{\delta_{e}} \delta_{e}+C_{L}^{\delta_{c}} \delta_{c}+C_{L}^{0}+C_{L}^{\eta} \eta \\
=4.6773 \alpha+0.76224 \delta_{e}+0.7408 \delta_{c}-0.0018714 \\
+4.249 * 10^{-2} \eta_{1}+0.01104 * \eta_{2}+1.785 * 10^{-2} \eta_{3} \\
M=z_{T} T+\bar{q} S \bar{c} C_{M}, \\
C_{M}=C_{M}^{\alpha^{2}} \alpha^{2}+C_{M}^{\alpha} \alpha+C_{M}^{\delta_{e}} \delta_{e}+C_{M}^{\delta_{c}} \delta_{c}+C_{M}^{0}+C_{M}^{\eta} \eta \\
=6.2926 \alpha^{2}+2.1335 \alpha+0.1986 \delta_{e}+0.23048 \delta_{c}+0.18979 \\
+2.569 * 10^{-2} \eta_{1}-0.3746 \eta_{2}+5.449 * 10^{-2} \eta_{3}
\end{gathered}
$$

And $V$ is speed, $\gamma_{\text {is }}$ the speed angle, $\alpha$ is attack angle, $Q_{\text {is }}$ the attitude angle speed, $h_{\text {is }}$ the height. $\phi$ is the oil supplying factor, $\delta_{c}$ is the duck wing and $\delta_{e}$ is the lift rudder.

\section{Simulation settings of free flying}

In order to testify the rightness of the model of hypersonic aircraft, choose a initial height as $h_{0}=85000 * 0.3048$, initial speed as $V_{0}=7846 * 0.3048$, initial attack angle as $\alpha_{0}=0.0174$, and other initial state as: $\gamma_{0}=0, q_{0}=0, \eta_{10}=0.4588 * 0.3048 * 14.59, \eta_{20}=-0.08726 * 0.3048 * 14.59$, $\eta_{30}=-0.03671 * 0.3048 * 14.59$, and set the oil supplying law as constant as $\phi=\phi_{c}=0.1$, and choose the control as $\delta_{c}=\delta_{e}=0$, then the constant attack angle flying test can be done by numerical simulation and simulation time is set as 0.5 second.

\section{Numerical Simulation and result analysis}

With above model and air coefficients and set initial condition as above paragraph and write a program with $m$ language in Matlab software, then the simulation can be done and simulation results can be shown as following figures. 


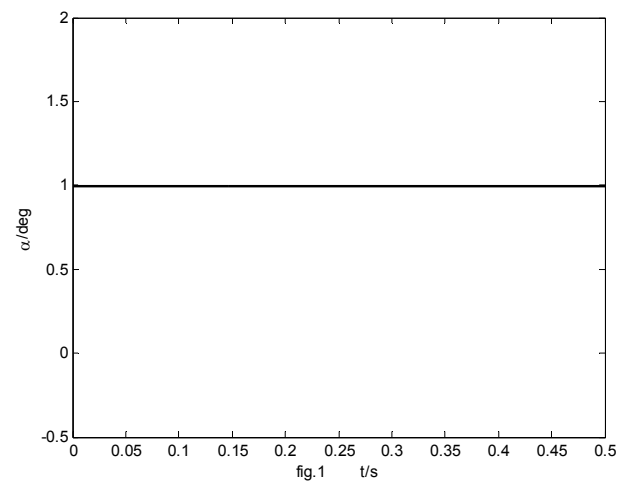

Fig 1 The curve of attack angle

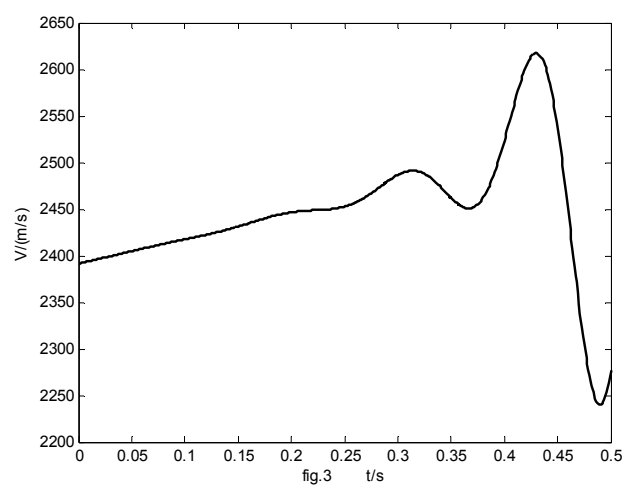

Fig 3 The curve of speed

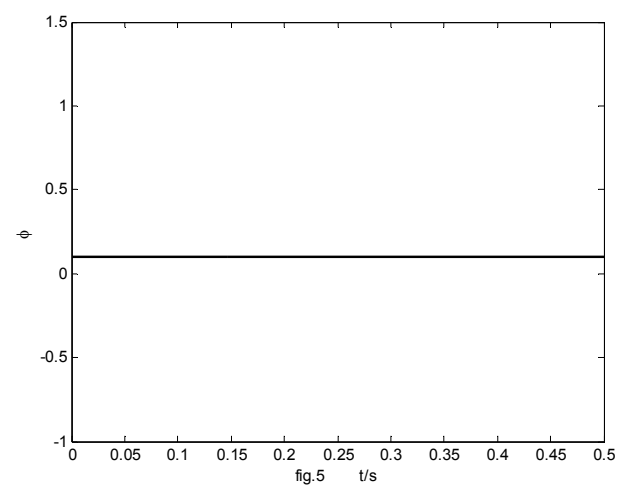

Fig 5 The curve of oil supplying factor

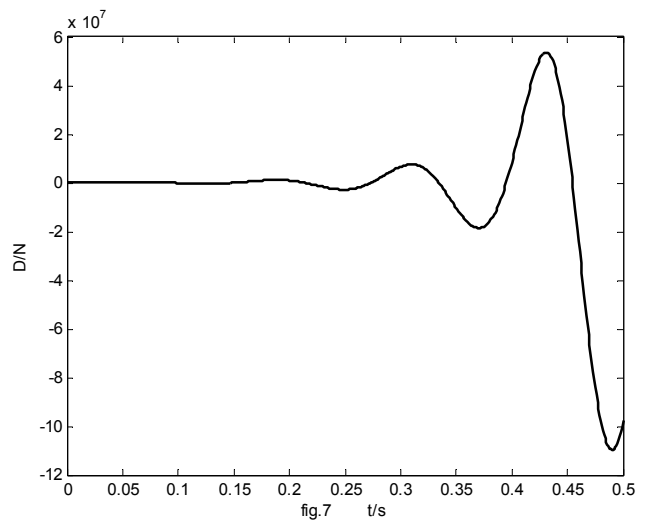

Fig 7 The curve of resistance

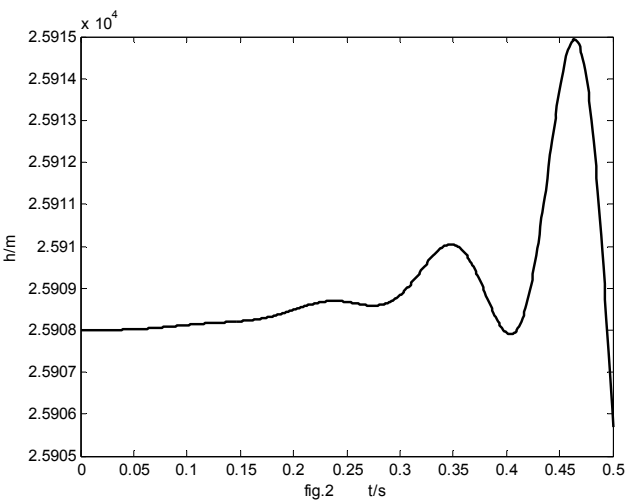

Fig 2 The curve of the height

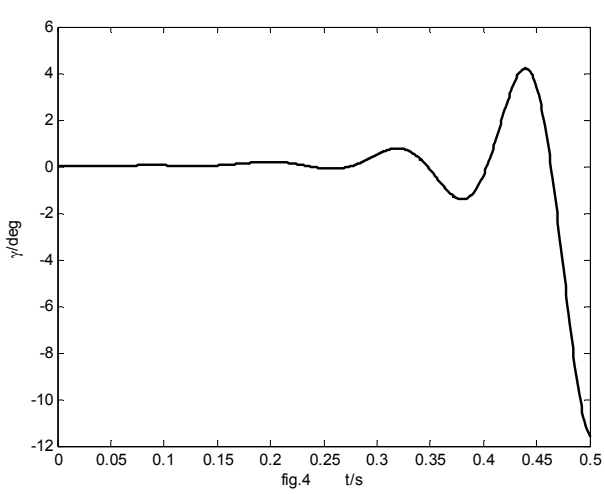

Fig 4 The curve of speed angle

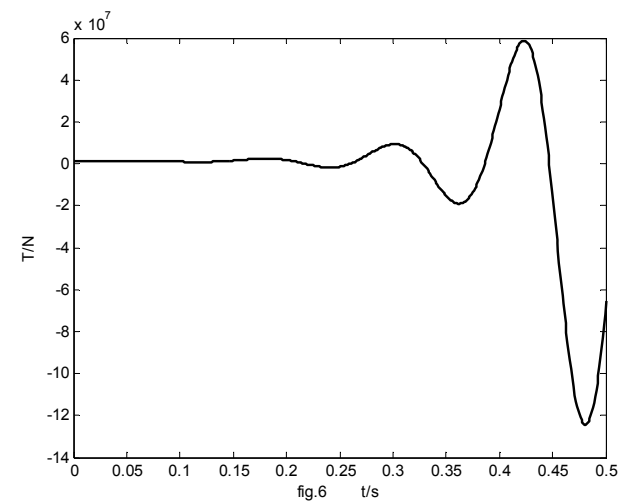

Fig 6 The curve of thrust

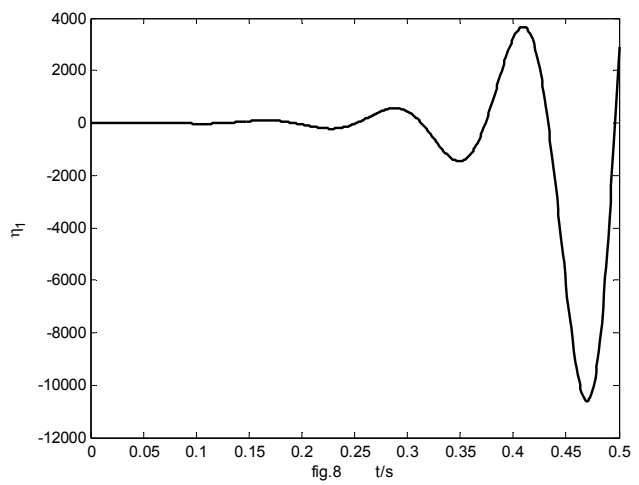

Fig 8 The state of first elastic shape 


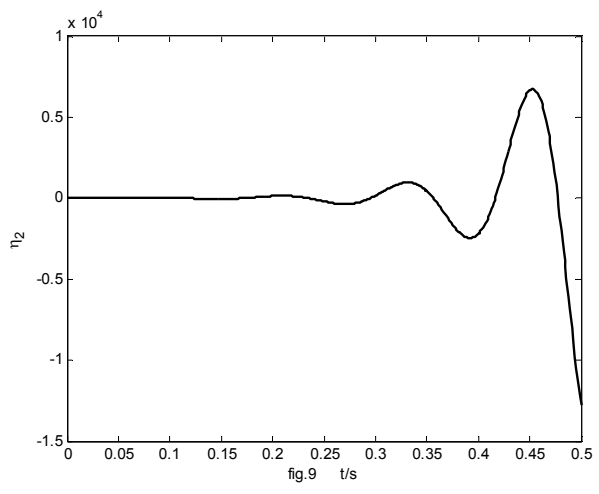

Fig 9 The state of second elastic shape

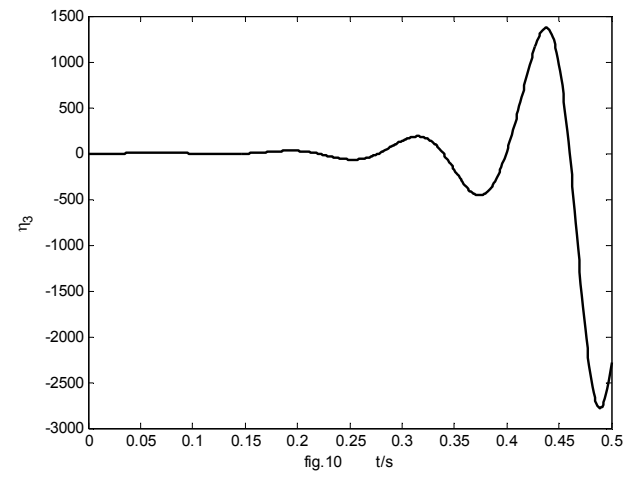

Fig 10 The state of third elastic shape

It can be seen that trajectory angle has greater divergence degree in the 0.5 seconds flight of fixed attack angle. It is caused by the elastic deformation, while this case will not happen in the low speed missile. The height and trajectory inclination are diverging slowly in the fixed attack angle flight of low-speed missile.

\section{Conclusion}

It can be found that $0.5 \mathrm{~s}$ for a given attack angle flight is basically stable from the above attack angle, altitude, speed, trajectory angle, engine throttle opening, thrust, resistance, elasticity curve shape. It verifies that the hypersonic vehicle model is reasonable. The basic purpose of the preliminary validation model is achieved. But diverging of superb aircraft elastic body shape is faster, leading to the surge in its force, so that the gesture diverges quickly, and this is the essence different with the traditional speed aircraft. Its posture and height can keep stable flying more than $2 \mathrm{~s}$ within a certain range when attack angle of traditions supersonic or subsonic aircraft is given.

\section{References}

[1]Wang J L, Sundararajan N. A nonlinear flight controller design for aircraft[J].Control Eng.Practice, 1995, 3(6): 813-825.

[2]A. E. Finzi, M. Lavagna, A. Di Gregorio, Atmospheric re-entry trajectory tracking and control for an unmanned space vehicle with a Lyapunov approach [A], In: AIAA Guidance, Navigation, and Control Conference and Exhibit [C], Austin: AIAA, 2003-5441

[3]Sigthorsson D O, Serranni, Yurkovich S, et a1. Tracking control for an overactuated hypersonic air-breathing vehicle with steady state constraints, In: AIA A Guidance, Navigation,and Control Conference and Exhibit,Keystone, USA, AIAA 2006-6558: 1-17.

[4]Vaddi S S, Sengupta P. Controller design for hypersonic vehicles accommodating nonlinear state and control constraints, In: AIAA Guidance, Navigation, and Control Conference Chicago, USA, AIAA 2009-6286: 1-19.

[5] Reiman S E, Dillon C H, Lee H P, et a1. Robust adaptive reconfigumble control for ahypersonic cruise vehicle, In: AIA A Guidance,Navigation, and Control Conference, Chicago, USA, AIA A 2009-6185: 1. 10.

[6]Christopher I. Marrison. synthesis of robust control systems for a hypersonic aircraft decision and contro1. IEEE, 1994。4:3324-3329

[7]E. mooij. linear quadratic regulator reentry control-performance assessment using a taguchi approach . AIAA, international space planes and hypersonic systems and technologies conference. 1998: 665-677.

[8]Kevin. P. Groves, Andrea. Serranti, Stephen. Yurkovich. Anti-Windup Control for an Air-breathing Hypersonic Vehicle Model. AIAA, 2006-6557: 1-14. 